\title{
Acoustic saturation in bubbly cavitating flow adjacent to an oscillating wall
}

\author{
T. Colonius, ${ }^{\text {a) }}$ F. d'Auria, and C. E. Brennen \\ Division of Engineering and Applied Science, California Institute of Technology, Pasadena, California 91125
}

(Received 2 August 1999; accepted 26 July 2000)

\begin{abstract}
Bubbly cavitating flow generated by the normal oscillation of a wall bounding a semi-infinite domain of fluid is computed using a continuum two-phase flow model. Bubble dynamics are computed, on the microscale, using the Rayleigh-Plesset equation. A Lagrangian finite volume scheme and implicit adaptive time marching are employed to accurately resolve bubbly shock waves and other steep gradients in the flow. The one-dimensional, unsteady computations show that when the wall oscillation frequency is much smaller than the bubble natural frequency, the power radiated away from the wall is limited by an acoustic saturation effect (the radiated power becomes independent of the amplitude of vibration), which is similar to that found in a pure gas. That is, for large enough vibration amplitude, nonlinear steepening of the generated waves leads to shocking of the wave train, and the dissipation associated with the jump conditions across each shock limits the radiated power. In the model, damping of the bubble volume oscillations is restricted to a simple "effective" viscosity. For wall oscillation frequency less than the bubble natural frequency, the saturation amplitude of the radiated field is nearly independent of any specific damping mechanism. Finally, implications for noise radiation from cavitating flows are discussed. (c) 2000 American Institute of Physics. [S1070-6631(00)00511-0]
\end{abstract}

\section{INTRODUCTION}

Cavitation leads to the production of noise, shock waves, and a host of flow instabilities. These, in turn, lead to damage of nearby solid surfaces, to radiated noise, and to degradation of the performance of devices. Previous investigators have identified the violent collapse of clouds of cavitation bubbles as a possible mechanism for noise generation and material damage. The violent collapse of the cloud leads to the production of intense bubbly shock waves, and may be related to other instabilities of the flow. Aside from the important problem of cavitation on propellers, there are a variety of applications where the nonlinear dynamics of bubbles play an important role. Experiments in these flows are often difficult, and therefore computational modeling is important not only for prediction, but also as a means to study the flow physics.

We report here computations of the bubbly cavitating flow caused by normal oscillation of a plane rigid wall adjacent to a semi-infinite domain of fluid. In a companion study, a similar methodology is applied to quasi-one-dimensional flows through de Laval nozzles. ${ }^{1}$ The computations are carried out for a cavitating flow model similar to the one originally proposed by van Wijngaarden. ${ }^{2}$ The essence of his approach, discussed in more detail in Sec. II, is to couple the Rayleigh-Plesset equation for the dynamics of spherical bubbles, on the microscale, to the equations for a continuum two phase flow on the macroscale. A significant body of literature now exists which uses similar modeling to explore the linearized dynamics of clouds of bubbles. ${ }^{3-7}$ Kumar and

\footnotetext{
a) Author to whom all correspondence should be addressed; electronic mail: colonius@caltech.edu
}

Brennen $^{8-10}$ have also explored weakly nonlinear solutions.

Fewer nonlinear solutions (which must be solved numerically) have been presented in the literature. Of these, methods which couple dynamic equations for the bubble microscale should be distinguished from methods which introduce an algebraic equation of state to relate mixture density to pressure. While the results of the latter (e.g., Refs. 11 and 12) may be of qualitative value, they imply a quasistatic situation which is inappropriate to most bubbly cavitating flows, which are inherently unsteady. Studies including dynamic (e.g., Rayleigh-Plesset) models for the bubbly microscale, include the numerical investigations of shock wave propagation in fluids with noncondensible gas bubbles, ${ }^{13,14}$ cavitation bubbles, ${ }^{15,16}$ and the dynamics of spherically symmetric clouds of bubbles, ${ }^{6,17-20}$ and the flow on a hydrofoil section. ${ }^{21}$ The Kubota et al. study, while most geometrically complex, involved an ad hoc limitation that bubbles were not permitted to collapse below their original nuclei size, which excludes the formation of the large pressure perturbations and shock waves which are an important part of cloud cavitation. Wang and Brennen ${ }^{18-20}$ highlight the role of inwardly propagating shock waves during the collapse of a cloud. Geometric focusing leads to large local pressures and suggests the potential for noise and damage.

In the present paper we investigate the simple cavitating flow caused by an oscillating wall. The motivation for studying the present configuration is twofold. First, the development of accurate numerical methods for bubbly cavitating flows is difficult, and it is useful to establish benchmark solutions to relatively simple problems where the accuracy of the numerics can be checked. Second, though the geometry is kept simple and restricted to one spatial dimension, the flow may be thought to represent, to first order, the flow in 
vibratory cavitation-damage-assessment devices. An important restriction of the present results is that an ad hoc, but computationally fast, model is used for damping the bubble radial motion. We argue in Sec. III B that the specific damping mechanisms are, for wall oscillation frequency much smaller than the bubble natural frequency, largely irrelevant to the overall dynamics of the mixture.

The paper is organized as follows. In Sec. II, the continuum bubbly flow model and its numerical implementation are discussed. An accurate nonreflecting boundary condition, which allows the semi-infinite domain to be truncated to a finite strip, is developed in Sec. II B. A Lagrangian finite volume scheme is derived in Sec. II C and validation is given in Sec. II D. Results are presented in Sec. III. In Sec. III C, an analytical expression for the radiated pressure waves, and their saturation, is developed in the low frequency limit. Concluding comments are placed in Sec. IV.

\section{THE MODEL AND NUMERICAL METHOD}

\section{A. Physical modeling}

We consider a continuum bubbly flow model. ${ }^{2,3,22,23}$ The equations and simplifying assumptions and their justification are briefly described here; the original references should be consulted for more detailed discussion of the derivation.

The essence of the model is to couple conventional continuity and momentum equations for a compressible liquidvapor mixture to a Rayleigh-Plesset equation for the bubble dynamics which provides the necessary relation connecting the local pressure with the bubble size and therefore the local mixture density. The principle modeling assumptions are that bubbles are spherical and that typical length scales associated with fluctuations in the mixture are large compared to the typical bubble radius. The mixture is further assumed to be dilute (low void fraction), to initially contain a large number of nuclei (heterogeneous nucleation), and fusion and fission of bubbles are not permitted. Moreover, the liquid phase is incompressible.

Further assumptions are that relative motion between the phases can be neglected, and liquid compressibility effects can be neglected in the Rayleigh-Plesset equation. These latter assumptions have been shown to have minimal impact on the linearized dynamics of spherical bubbles. ${ }^{3-5,22}$

Damping of the bubble radial motion, which in reality is governed by a complex interaction of diffusive and thermodynamic effects, is modeled with a simple "effective" damping, and the further assumption that any noncondensable gas in the bubbles is fully mixed and undergoes isentropic compression and expansion. The use of a polytropic (and isentropic) exponent is not strictly valid for nonlinear oscillations of bubbles. ${ }^{24}$ Far from the wall, oscillations are nearly linear and, for low frequency, isothermal behavior is probably more realistic. Near the wall, however, bubble collapse is violent and nonlinear, and adiabatic behavior seems more appropriate (especially considering the internal motions of the gas that would exist if spherical symmetry is lost). To avoid solution of unsteady radial diffusion equations for mass, momentum, and energy for the bubble contents at each position in the mixture, we choose for simplicity to use the isentropic approximation for all bubbles, along with the simple "effective" damping discussed previously. The impact of this simplified model for the damping is further discussed in Sec. III B.

These assumptions lead to macroscale conservation equations for mass and momentum of the bubbly mixture:

$$
\begin{aligned}
& \frac{D \rho}{D t}+\rho \frac{\partial u_{i}}{\partial x_{i}}=0, \\
& \frac{D u_{j}}{D t}+\frac{1}{\rho} \frac{\partial p}{\partial x_{j}}=0 .
\end{aligned}
$$

Note that viscous terms and gravity have been neglected in the momentum equation.

On the microscale, the typical bubble radius, $R(x, t)$, is governed by the Rayleigh-Plesset equation:

$$
\begin{aligned}
& R \frac{D^{2}(R)}{D t^{2}}+\frac{3}{2}\left(\frac{D R}{D t}\right)^{2}+\delta_{D} \frac{1}{R} \frac{D R}{D t}+\frac{2}{\mathrm{We}}\left(R^{-1}-R^{-3 \gamma}\right) \\
& +\frac{\sigma}{2}\left(1-R^{-3 \gamma}\right)+P=0 .
\end{aligned}
$$

The system of equations is closed by noting that the above-mentioned assumptions lead to a relation between the mixture density and bubble radius:

$$
\rho=\left(1+\frac{\alpha_{0} R^{3}}{1-\alpha_{0}}\right)^{-1} .
$$

In Eqs. (1)-(4) the mixture density, $\rho$, is made dimensionless by the constant liquid density, $\rho_{L}$. The initial void fraction is denoted by $\alpha_{0}$. Lengths, $x_{i}$, and the bubble radius, $R$, are normalized by an equilibrium bubble radius, $R_{0}$, and the mixture velocity, $u_{i}$, is normalized by the equilibrium bubble natural frequency, $\omega_{0}$, times the equilibrium bubble radius. The pressure, $P$, is measured relative to its equilibrium value, $p_{0}$, and normalized by $\rho_{L} R_{0}^{2} \omega_{0}^{2}$. Time, $t$, is normalized by $1 / \omega_{0}$. Moreover, $\sigma$ is the cavitation number, defined as $\left(p_{0}-p_{v}\right) / \frac{1}{2} \rho_{L} R_{0}^{2} \omega_{0}^{2}$, where $p_{v}$ is the vapor pressure. The ratio of specific heats for a noncondensable gas in the bubble is $\gamma$. The effective damping for spherical bubbles is denoted by $\delta_{D}$ and is discussed in detail in Sec. III B. The Weber number is given by $\mathrm{We}=\rho_{L} R_{0}^{3} \omega_{0}^{2} / S$, where $S$ is the (constant) surface tension. We note that these equations may be derived using a detailed ensemble phase averaging technique, as describe by Zhang and Prosperetti. ${ }^{25}$

As a result of the way in which the equations have been nondimensionalized, the values $R=1, P=0, u=0$, and $\rho$ $=1-\alpha_{0}$ constitute a steady solution of Eq. (3), and this is referred to as bubble equilibrium. For a slight disturbance from this equilibrium state, bubbles will oscillate with their natural frequency, $\omega_{0}$. Since the equations have been normalized by $\omega_{0}$, the expression for the natural frequency becomes

$$
\frac{2}{\mathrm{We}}(3 \gamma-1)+\frac{3 \gamma \sigma}{2}=1 .
$$

In what follows we consider $\mathrm{We} \gg 1$ and $\gamma=1.4$, and thus

$\sigma \approx 0.475$. 
Moreover, for convenience we confine attention to a fixed equilibrium void fraction, $\alpha$, of $1 \%$.

\section{B. Boundary conditions}

We consider a semi-infinite region bounded by a flat moving wall. The boundary condition for the wall is that the fluid velocity normal to the wall is equal to the wall velocity. In the following we specifically consider sinusoidal motion of the wall given by

$$
u_{w}\left(x_{w}, t\right)=A \sin (2 \pi(t / T)) .
$$

This introduces two additional nondimensional parameters, the amplitude, $A$ (relative to $R_{0} \omega_{0}$, and the frequency of wall oscillation, $\omega_{w}$, which is made dimensionless with $\omega_{0}$.

Often a semi-infinite domain can be treated numerically, at least for incompressible flow problems, by mapping the infinite domain to a finite computational one. For compressible flow problems which involve acoustic waves that propagate to infinity and decay only slowly (or not at all in the case of one-dimensional inviscid flow), domain mappings are problematic since the waves become more and more poorly resolved as they propagate through the highly stretched mesh near infinity. In these types of problems, therefore, the computational domain is usually truncated at a finite location, and an artificial boundary condition is imposed.

To pose an artificial boundary condition, it is necessary to make additional assumptions about the flow outside the region of interest. Considering the one-dimensional flow next to the vibrating wall, we suppose that the bubbly flow is semi-infinite and impose a nonreflecting boundary condition at the edge of the truncated domain. The nonreflecting boundary condition (approximately) eliminates any incoming wave at the edge of the computational domain.

The development of nonreflecting boundary conditions, even for hyperbolic (nondispersive) problems, is difficult in the general multidimensional case. The reader is referred to recent review papers ${ }^{26,27}$ for a detailed discussion of the important issues. For linear one-dimensional hyperbolic systems an exact nonreflecting boundary condition may be obtained by decomposing the solution into a set of decoupled (characteristic) waves. In the present case, the waves are nonlinear and dispersive. However, small amplitude (linearized) disturbances with frequency $\omega$ and wave number $k$ will propagate at a speed

$$
c=\omega / k= \pm\left(\frac{1}{3 \alpha_{0}\left(1-\alpha_{0}\right)}\left(1-\omega^{2}-i \omega \delta_{D}\right)\right)^{1 / 2} .
$$

Equation (8) follows from the Fourier transform of the linearized versions of Eqs. (1)-(4) (e.g., Ref. 28). Note that $\omega$ is normalized by $\omega_{0}, k$ is normalized by $1 / R_{0}$, and $c$ is normalized by $R_{0} \omega_{0}$.

Furthermore, for one-dimensional small amplitude disturbances, it can be shown that the Fourier amplitudes of the velocity and pressure, $\hat{u}$ and $\hat{p}$, respectively, are related by

$$
\hat{p}=Z(\omega)^{ \pm} \hat{u},
$$

where $Z(\omega)^{ \pm}$is the "acoustic impedance," given by
$Z(\omega)^{ \pm}= \pm\left(1-\alpha_{0}\right) c= \pm\left(\frac{\left(1-\alpha_{0}\right)}{3 \alpha_{0}}\left(\omega_{0}^{2}-1-i \omega \delta_{D}\right)\right)^{1 / 2}$,

where the positive and negative roots correspond to rightgoing and left-going waves (and positive and negative phase velocities), respectively. Thus at one boundary of the domain, a nonreflecting boundary condition is

$$
\hat{p}=Z^{+} \hat{u}+\hat{p}_{I},
$$

where we have added $\hat{p}_{I}$ to allow the possibility of arbitrarily specified incoming pressure disturbances. Equation (11) is nonlocal in time since it contains the square root of the frequency. If the frequency is further assumed to be small, then we can approximate Eq. (11) by

$$
p\left(x_{N}, t\right)=\rho_{0} c_{0} u\left(x_{N}, t\right)-\frac{\delta_{D} c_{0}}{2} \frac{\partial p}{\partial x}\left(x_{N}, t\right)+p_{I},
$$

where

$$
c_{0}=\omega_{0}\left(\frac{1}{3 \alpha_{0}\left(1-\alpha_{0}\right)}\right)^{1 / 2} .
$$

The efficacy of these nonreflecting boundary conditions is discussed in Sec. II D.

\section{A Lagrangian finite volume scheme}

It is not trivial to obtain an accurate and efficient numerical solution of flow equations coupled to highly nonlinear bubble dynamics. In the present work we begin by deriving a numerical method for one-dimensional flow. The development of accurate multidimensional schemes will be pursued in future publications.

Equations (1)-(4) are integrated using a onedimensional Lagrangian finite volume scheme in which each control volume face moves at the local fluid velocity. The Lagrangian framework is convenient for two reasons: First, it facilitates the application of the boundary condition at the moving wall, and second, it allows the Rayleigh-Plesset equation to be integrated (for a particular Lagrangian element) as an ordinary differential equation (ODE). The method discretely conserves both mass and momentum, and thus appropriately preserves "jump" conditions across bubbly shock waves (which, unlike gas dynamic shocks, have finite thickness with an internal structure dictated by inertial effects).

Consider a one-dimensional space divided into a collection of $N$ control volumes. Integrating Eqs. (1) and (2) over the control volumes we obtain, for $j=1,2, \ldots, N-1$ :

$$
\begin{aligned}
& \frac{d}{d t} \int_{x_{j}}^{x_{j+1}} \rho d x=0, \\
& \frac{d}{d t} \int_{x_{j}}^{x_{j+1}} \rho u d x=P_{j}-P_{j+1} .
\end{aligned}
$$

Equations (13) and (14) describe the rate of change of the total mass and momentum in the $j$ th control volume. Each of the faces $(j=1,2, \ldots, N)$ of the control volume moves with the local fluid velocity and therefore 


$$
\frac{d x_{j}}{d t}=u_{j}
$$

where $u_{j}$ is shorthand for $u\left(x_{j}(t), t\right)$. Also, Eq. (3) can be split into two first-order equations at each face:

$$
\begin{aligned}
& \frac{d R_{j} V_{j}}{d t}+G_{j}+P_{j}=0, \\
& \frac{d R_{j}}{d t}-V_{j}=0,
\end{aligned}
$$

where

$$
G_{j}=\frac{V_{j}^{2}}{2}+\delta_{D} R_{j}^{-1} V_{j}+\frac{2}{\mathrm{We}}\left(R_{j}^{-1}-R_{j}^{-3 k}\right)+\frac{\sigma}{2}\left(1-R_{j}^{-3 k}\right) .
$$

Finally, the density and bubble radius at the faces are related by

$$
\rho_{j}=\left(1+\frac{\alpha_{0} R_{j}^{3}}{1-\alpha_{0}}\right)^{-1}
$$

To integrate this system of (as yet exact) equations, it remains to approximate the integrals in Eqs. (13) and (14). A second-order approximation is used:

$$
\int_{x_{j}}^{x_{j+1}} f d x=\frac{\Delta x_{j}}{2}\left(f_{j}+f_{j+1}\right)+O\left(\Delta^{3}\right),
$$

where $\Delta x_{j}=x_{j+1}-x_{j}$, and where $f$ is any of $R_{j} V_{j}, R_{j}$, or $M_{j}$.

Equations (13)-(19) are 6N-2 ODEs for $6 N$ unknowns ( $\rho_{j}, R_{j}, V_{j}, x_{j}, u_{j}$, and $P_{j}$ at the edges of the control volumes, $j=1,2, \ldots, N)$. Specifying two boundary conditions closes the system. At the moving solid wall the velocity $u_{1}$ is prescribed. The approximate nonreflecting boundary condition derived previously [Eq. (12)] is applied at the other end of the computational domain.

These equations are solved in the Lagrangian coordinate system. Note that, depending on the solution, the control volumes could become very small or very large. If they become large then the trapezoidal rule given in Eq. (20) may not be accurate. In that case it may be necessary to remesh the computation by interpolating the Lagrangian quantities back to a regular grid.

An interesting feature of the discretized equations is that an explicit time marching of the equations will not conserve mass precisely. For explicit time marching, Eqs. (15) and (17) give $x_{j}$ and $R_{j}$ at the new time level. Equation (19) then gives the density at the new time level, and so in general, Eq. (13) cannot be satisfied at the new time level. This may be related to difficulties previous investigators have encountered in solving similar equations with explicit schemes. ${ }^{6,29}$

For this reason (and the additional advantage of handling stiffness) an implicit time marching scheme is used. The method chosen is a Richardson extrapolation method based on the implicit Euler method. For a given time step, a series of predictions are made for the solution at the new time level based on different numbers of subdivisions of the time interval. The series of predictions is then used to extrapolate to the limit of zero time step, and to provide an error estimate for the integration. The overall time step is adjusted based on the number of subdivisions and the error estimate. The details of the scheme are as given by Hairer and Wanner. ${ }^{30}$ Numerical experiments showed that the extrapolation method is much more efficient than first- and second-order implicit schemes.

The basic time advancement of the extrapolation method is chosen to be the implicit Euler method. Using this integration scheme on Eqs. (13)-(19) and going through the algebra, we can establish $N$ equations of the form

$$
F_{j}\left(R_{j-1}^{n+1}, R_{j}^{n+1}, R_{j+1}^{n+1}\right)=0, \quad j=1,2, \ldots, N,
$$

which contain $N$ unknowns, $R_{j}^{n+1}$, for $j=1,2, \ldots, N$. In each equation $F_{j}$, various parameters of the problem also appear as well as the fields from previous time levels. To solve the nonlinear equations we use Newton's method. In the present case, the Jacobian matrix is tridiagonal in form and so Newton iterations are rapidly solved.

Finally, the stability of the above-mentioned numerical scheme was analyzed using the von Neumann method (e.g., Ref. 31) for the linearization of Eqs. (13)-(19) about the equilibrium flow $(R=1)$. The resulting ODE is, in the semidiscrete limit, inherently stable, and therefore A-stable implicit schemes will also be stable. Note that the extrapolated schemes used here are not A-stable, but only nearly so, and they can be unstable for eigenvalues which lie near the imaginary axis. ${ }^{30}$ In practice we have found that our scheme is stable even for large time steps, and even in the presence of significant nonlinearity.

\section{Code validation}

Calculation of steadily propagating bubbly shock waves provides a useful test case for the numerical method, as their structure can be computed, in a stationary reference frame, by solving an ODE. We instigate a shock wave in the computational domain by specifying a pressure increase at the left boundary, in the form of an incident wave whose pressure is given by

$$
p_{I}=\frac{p_{A}}{2}\left(1+\tanh \left(\frac{t}{T_{f}}\right)\right) \text {. }
$$

The parameter $T_{f}$ controls the time scale over which the pressure jump is accomplished. The pressure rise begins propagating to the left through the domain at the sonic speed given earlier in Eq. (8). As it propagates, nonlinearity cause the wave front to steepen and accelerate, and eventually a bubbly shock wave is formed. It (eventually) propagates to the left at a (constant) speed, $u_{1}$. To test the accuracy of the code, we compare in Fig. 1 the shock wave structure from the present unsteady code with the steady bubbly shock wave solution obtained by solving Eq. (6.72) of Brennen. ${ }^{28}$ Note that the steady equation is an ODE which must also be solved numerically and requires an initial position and rate of change of bubble radius with position which are taken from the unsteady numerical solution. The figures show excellent agreement between the two independent solutions. Apparently as the resolution of the unsteady solution is increased, 

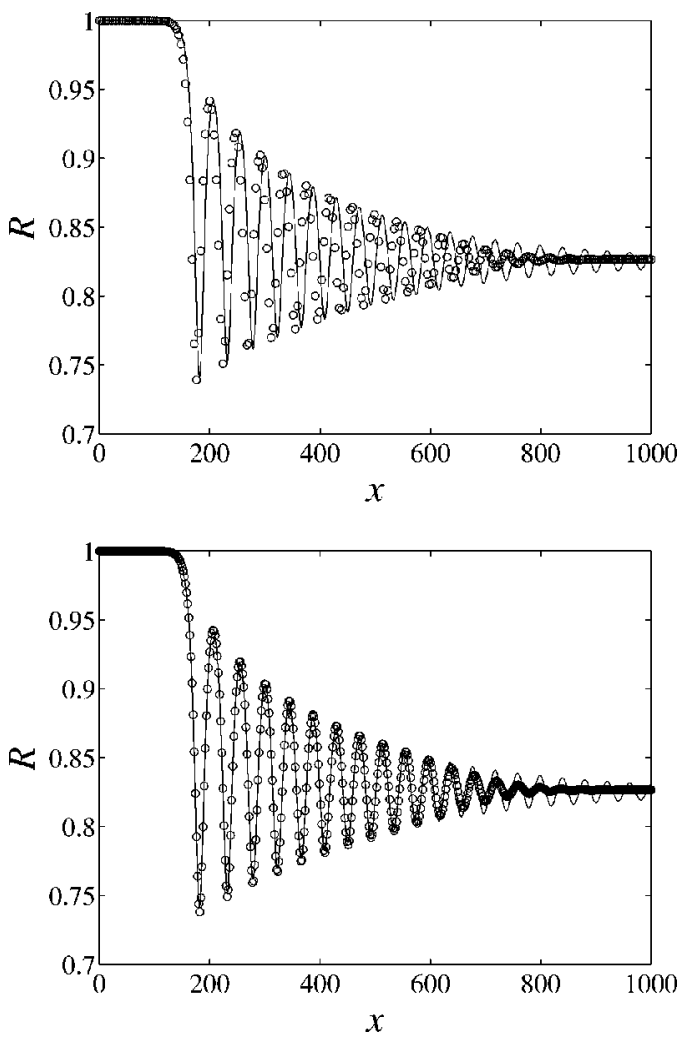

FIG. 1. Comparison of numerically calculated unsteady shock wave $(\bigcirc)$ and a steady shock (-) given by the solution of Eq. (6.72) of Brennen (1995). The various parameters (made nondimensional as discussed previously) are: $\mathrm{We}=1870, \delta_{D}=0.04, \alpha_{0}=0.01, t_{0}=100, T_{f}=25, \sigma=0.475, k=1.4\left(\omega_{0}\right.$ =1). (a) $\Delta x_{j}$ is initially 4 for each cell; (b) $\Delta x_{j}$ is initially 2 . These values do not change significantly during the course of the shock propagation.

the phase error between the two solutions is decreased. There is a slightly exaggerated decay of the subsequent rebounds and collapses in the numerical solution due to numerical dissipation. It should be noted that the present unsteady shock has propagated a substantial distance by the time the comparison is made. One would expect further dissipation of the collapses and rebounds to take place upon further propagation.

\section{E. Test of nonreflecting boundary condition}

A series of computations were also performed to test the efficacy of the nonreflecting boundary condition. An incoming wave was generated at the nonreflecting boundary by specifying

$$
p_{I}=p_{A} \exp \left(-(t / T)^{2}\right) .
$$

If $T$ is large enough, the energy of the wave is restricted to low frequencies, and, for small amplitude, it should propagate nondispersively at a speed, $c$, given by Eq. (8). The wave propagates first in the negative $x$ direction, reflects from the solid wall, propagates in the positive $x$ direction, and eventually passes through the nonreflecting boundary. Because the boundary condition is approximate, some fraction of the wave energy is reflected back, and the process continues until there is no energy (or rather until there is nothing but accumulated numerical error) left in the domain.

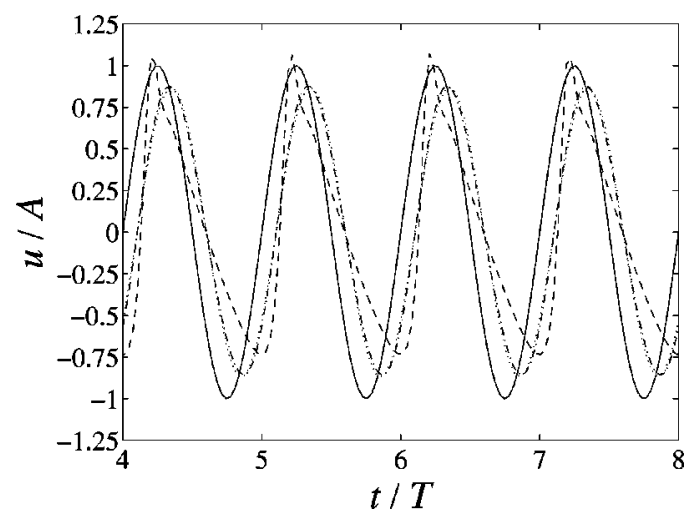

FIG. 2. Nonlinear steepening of the waves for $\mathrm{We}=1870, \delta_{D}=0.4, \alpha_{0}$ $=0.01, \sigma=0.475$. A grid of 801 points is used on a domain 400 units wide (relative to the initial equilibrium bubble radius). Plotted are: The wall velocity (-) and the particle velocity at $x=400$ for $A=0.0001(\cdots), A$ $=0.001(-\cdot-)$, and $A=0.01(--)$. All velocities are normalized by the amplitude of the wall velocity, $A$.

A measure of the efficacy of the boundary condition is the history of total "acoustic energy," which is estimated simply as $E(t)=\sum_{j=1}^{N} p_{j}^{2}$. Tests show that for small amplitudes $\left[p_{A}=O\left(10^{-3}\right)\right.$ and smaller] the reflection coefficient (ratio of energy in the domain before and after passage of the pulse through the boundary) is about $0.0016 \%$. As the amplitude is increased the reflection coefficient increases owing to nonlinear effects which are not accounted for in the analysis. However, for $p_{A}$ as large as 0.05 the reflection coefficient increases only to $0.36 \%$, and clearly most of the energy is still absorbed by the boundary. Note that for these large amplitude disturbances the incident wave steepens and forms a bubbly shock, similar to that described previously.

\section{RESULTS}

\section{A. Low frequency vibration}

We begin by considering low frequency wall vibration, $\omega_{w} \ll 1$. In what follows, we set the effective damping coefficient, $\delta_{D}$, in the Rayleigh-Plesset equation to 0.4 . The effects of damping are discussed in Sec. III B.

For small enough amplitudes, nonlinear effects are absent and, for low enough frequency, the phase speed of pressure disturbances is real and constant (not a function frequency), according to Eq. (8). That is, wall vibration generates propagating disturbances that are only slightly attenuated by the damping in the Rayleigh-Plesset equation. For higher frequencies, again at sufficiently low amplitude, the phase speed in Eq. (8) is dispersive. Neglecting the small dissipation, the phase speed is purely imaginary for frequencies greater than $\omega_{0}$. In this case the wave motion is cut-off, with disturbances decaying exponentially with distance from the wall.

Plotted in Fig. 2 are time traces of the mixture velocity at a distance of $400 R_{0}$ from the wall, for $\omega_{w}=0.1$, and for relatively low amplitudes of wall vibration. In Fig. 2, the velocity has been normalized by the amplitude, which allows a wide range of amplitudes to be depicted on the same scale. This indicates nearly linear behavior. Note that the time axis 


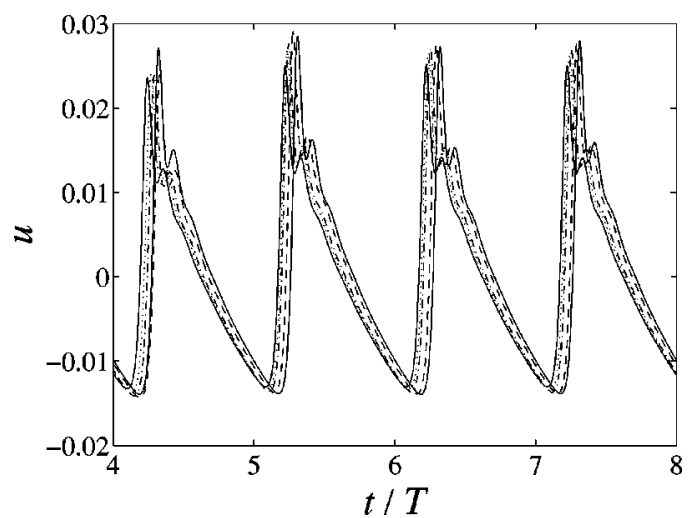

FIG. 3. Asymptotic shock structure for the same parameters as in Fig. 2 but with $A=0.05(-), A=0.075(\cdots), A=0.01(-\cdot-), A=0.2(--)$, and $A$ $=0.3(-)$. Note that the velocities are not normalized by $A$.

is scaled by the period of wall oscillation, and is only plotted after initial transients have died out; the wave train is periodic. For the largest amplitude in the plot, $A=0.01$, nonlinear steepening of the waves is evident.

For still higher amplitudes, the compressions steepen into shock waves, and the waves take the approximate form of a periodic train of $N$ waves. This is illustrated in Fig. 3, which is similar to Fig. 2, but with higher amplitudes. Note that in Fig. 3, the velocity is not scaled with the amplitude of the wall velocity. Clearly a saturation of the radiated energy takes place. This is due to the presence of shocks which dissipate increasing amounts of energy as the amplitude is increased. The process is similar to the acoustic saturation phenomena, which is well known in gas dynamics. In fact, for low frequencies, the saturated wave form can be predicted analytically, which we demonstrate in Sec. III C.

Very near the wall, as the amplitude is increased, significant bubble growth (cavitation) occurs in a thin layer near the wall. The bubble size, $R(x, t)$, adjacent to the wall is shown as a function of time in Fig. 4. The response of the layer near the wall is reminiscent of the response of a single bubble to a harmonic pressure field.

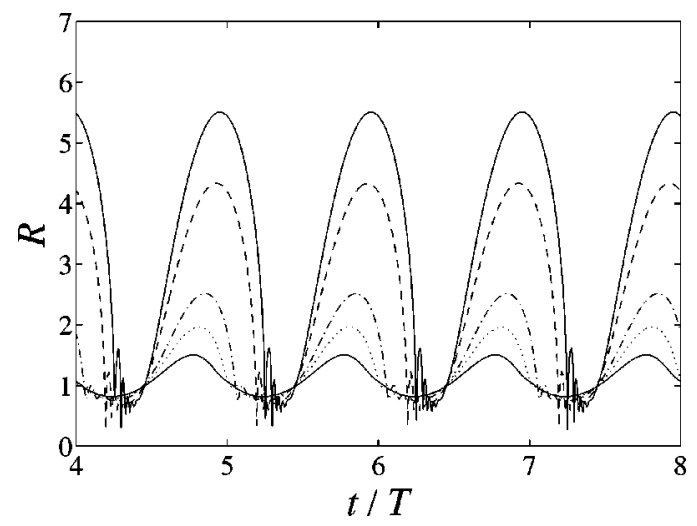

FIG. 4. Growth and collapse of bubble radius near wall (the same parameters and legend as in Fig. 3).

\section{B. The effective damping parameter}

In this section we discuss the limitations of the use of an effective damping parameter for volume oscillations of spherical bubbles. It has been recognized for many years that several different physical processes may contribute to this damping. Chapman and Plesset ${ }^{32}$ provided a useful summary of the relative magnitudes of the contributions from viscous liquid effects, from acoustic radiation, and from thermal effects. More recent work has further quantified these processes in nonlinear calculations. For noncondensible gas bubbles, Watanabe and Prosperetti ${ }^{33}$ showed that the thermal exchange between bubble and liquid must be modeled by solving an unsteady radial heat diffusion equation for the bubble contents. Their results showed that this process was not well modeled by a single "effective" polytropic index. They were able to show that their model agreed with experiments for shocks that had time to broaden from an initial sharp front to a more diffuse profile. The issue is also discussed by Kameda et al. ${ }^{14}$ who also show a substantial impact of the thermal effects on the shock profile. The relative importance of thermal diffusion for vapor (produced by cavitation) or mixtures of vapor and noncondensible gas is not as clear as it is for bubbles consisting only of noncondensible gas, ${ }^{34}$ and detailed computations of shocks in such flows (including heat and mass diffusion effects) have not yet been attempted.

Another process which leads, on average, to the dissipation or "smoothing" of bubble oscillations is statistical variations in bubble size either spatially or in different realizations of the flow (or both). The basic process has been demonstrated in computations by Kameda et al. ${ }^{14}$ and by Wang, ${ }^{35}$ though neither computational model included all effects consistent with statistical variations in the low void fraction approximation, as can be seen by comparing their models with the rigorous ensemble phase-averaging process developed by Zhang and Prosperetti. ${ }^{25}$

A final uncertainty in real cavitating bubbly flows is the likelihood that the fission of bubbles and other departures from sphericity, which often occurs in the first collapse, introduces additional (and perhaps dominant) dissipation that reduces the number and magnitude of the rebounds after the first collapse. Quantifying this additional attenuation mechanism presents a real challenge as yet not met.

In the present work, we have tried to account for all these effects in a computational fast, albeit crude, manner, by using a total "effective" viscosity in place of the physical liquid viscosity in the Rayleigh-Plesset equation. This simple approach has been used previously, for example, to match experimental results for the attenuation of small amplitude (linear) acoustic waves, though with mixed results. ${ }^{36}$

The limitations of this approach are now discussed. For the results presented in Sec. III A we have used $\delta_{D}=0.4$. Values of $\delta_{D}$ as large as 0.5 have been used to match theoretical and experimental predictions for the attenuation of acoustic waves in bubbly mixtures. ${ }^{36}$ Interestingly, for sufficiently low frequencies of wall vibration, it turns out that $\delta_{D} \approx 0.5$ represents a significant transition in the results presented in Sec. III A. To demonstrate this, the bubble radius at 


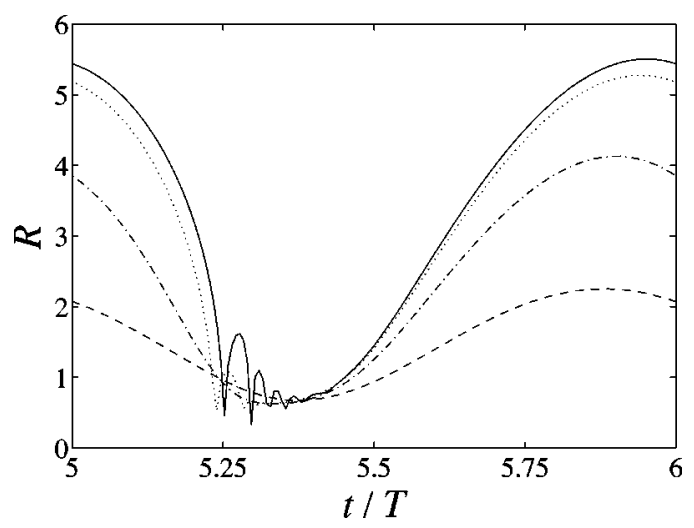

FIG. 5. Growth and collapse of bubble radius near wall for $A=0.3$ and $\delta_{D}=0.4(-), \delta_{D}=0.8(\cdots), \delta_{D}=4.0(-\cdot-)$, and $\delta_{D}=40.0(--)$.

the wall is plotted in Fig. 5 over one period, for a series of runs with $A=0.3$ but with differing values of $\delta_{D}$. For $\delta_{D}$ greater than 0.8 the maximum bubble radius begins to decrease significantly. For this large value of the damping there is no violent collapse and rebound of the cloud. For values below 0.8 the maximum bubble radius appears to saturate, and the only significant difference in the results is the number of collapses and rebounds during each cycle. For values of $\delta_{D}$ much less than 0.4 (not shown in the plot), the larger number of rebounds lead to much greater computational requirements for adequate resolution of both temporal and spatial features of the flow.

Thus, at low frequency, there appears to be an upper limit on the effective damping, beyond which the damping has a significant impact on the entire solution for the mixture. Below this value, varying the effective damping changes only the attenuation of the "ringing" of the bubbles following collapse. Such ringing may have an impact on the high frequency acoustic field produced by cavitating flows but it is evidently not of dynamic significance to the flow of the mixture. The detailed reason for this, as derived in Sec. III C, is that the dissipation associated with the shock jump conditions is much larger than, and independent of, the dissipation provided by any of the aforementioned processes. Thus it appears, for low frequency forcing, that it is relatively unimportant to model the detailed thermal processes in the bubble.

As the externally imposed frequency approaches the bubble natural frequency, it is not presently clear whether realistic results can be obtained with the effective damping. Indeed, computations we performed for high frequency oscillation showed that the collapse and rebound process is intimately related to the dynamics of the overall mixture, and there was no way to justify the lumping of all dissipative effects into a single effective damping.

\section{Analytical model of acoustic saturation}

As discussed in Sec. III A, large amplitude wall vibration, at low frequency, results in a saturation of the acoustic energy which is radiated away from the wall. In this section, we show that a model for the radiated wave form and its saturation pressure may be obtained in the limit of vanishing forcing frequency, even though the flow adjacent to the wall remains significantly modified by bubble dynamics (that is, the flow is not barotropic).

A well known result for plane waves generated by normal oscillation of a wall in a gas is acoustic saturation (see the discussion by Pierce, ${ }^{37}$ which contains the original references). Even in the limit of vanishing dissipation mechanisms (e.g., viscosity, heat conduction, etc.), acoustic energy is dissipated when compressions steepen into shocks. For shocks, the total amount of dissipation is, for small viscosity, independent of viscosity, dependent only on the "jump conditions" across the shock. Larger shocks dissipate more energy. Thus as the amplitude of vibration is increased, waves shock closer and closer to wall, and with greater shock strength. The pressure at a fixed distance from the wall, sufficiently far from the wall, then becomes independent of the amplitude of vibration.

A weak shock analysis (e.g., Ref. 37) shows that at a fixed value of $x$, the maximum pressure (over a cycle) is given (in dimensional form) by

$$
p_{\max }=\left\{\begin{array}{l}
p_{A} \quad \text { if } x<\frac{\pi \rho_{0} c_{0}^{3}}{2 \beta_{0} p_{A} \omega} \\
\frac{\pi p_{A}}{1+p_{A}\left(\frac{x \beta_{0} \omega}{\rho_{0} c^{3}}\right)} \quad \text { if } x>\frac{3 \pi \rho_{0} c_{0}^{3}}{\beta_{0} p_{A} \omega},
\end{array}\right.
$$

where $p_{A}$ is the amplitude of pressure oscillation at the wall, $\rho_{0}$ and $c_{0}$ are the ambient density and speed of sound, $\omega$ is the frequency of oscillation, and $\beta_{0}$ is a thermodynamic quantity given, for a perfect gas, by

$$
\beta_{0}=1+\rho_{0} c_{0}\left(\frac{\partial c}{\partial p}\right)_{0},
$$

where the partial derivative is at fixed entropy and evaluated at ambient conditions. The quantity $\beta_{0}$ is a measure of the nonlinear steepening of the waves, and stems from two effects. The first is that, for compression, the wave's particle velocity enhances its propagation speed. The second is that the speed of sound is increased by compression. Note that for a perfect gas, the first effect is the dominant one, and that $\beta_{0}=(\gamma+1) / 2$.

To apply Eq. (24) to a bubbly cavitating flow, we take the limit of zero frequency in Eq. (8) to obtain (reverting to dimensional quantities)

$$
c_{0}^{2}=\frac{R_{0}^{2} \omega_{0}^{2}}{3 \alpha_{0}\left(1-\alpha_{0}\right)},
$$

where the subscript " 0 ", here refers to ambient conditions with bubble equilibrium. For large Weber number, we have, from Sec. II, that $\gamma \sigma=1 / 6$ and thus

$$
c_{0}^{2}=\frac{\gamma\left(p_{0}-p_{v}\right)}{\alpha_{0} \rho_{0}} .
$$

This can be differentiated with respect to $p_{0}$ to obtain $\beta_{0}$ :

$$
\beta_{0}=1+\frac{\gamma+1+2 \alpha_{0}}{2 \alpha_{0}\left(1-\alpha_{0}\right)},
$$




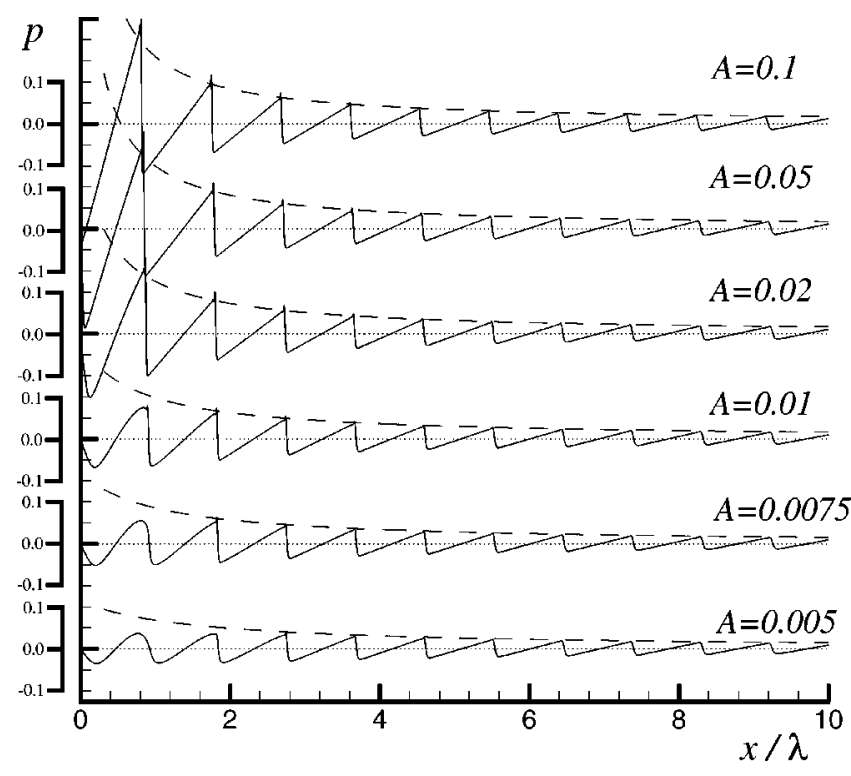

FIG. 6. Spatial evolution of the (nondimensional) pressure for wall oscillation at $0.02 \omega_{0}$ for several different amplitudes of oscillation and We $=1870, \delta_{D}=0.4, \alpha_{0}=0.01, \sigma=0.475$. The dashed lines are plots of Eq. (24).

where the expression $\rho_{0} \approx \rho_{L}\left(1-\alpha_{0}\right)$ has been used. For small $\alpha_{0}$ this becomes

$$
\beta_{0} \approx \frac{\gamma+1}{2 \alpha_{0}}
$$

which is identical to the result for a pure perfect gas, except that it is divided by the void fraction. The result is singular as the void fraction is decreased to zero, since the liquid has been considered as incompressible. Values of $\beta_{0}$ in a real pure liquid are on the order of 10 . It should be noted that larger values of $\beta$ imply that shocks are formed with a lower amplitude of vibration than would be necessary in a pure gas.

Equation (24) is compared in Fig. 6 to the computed pressure as a function of distance from the nominal wall position, for several amplitudes of wall vibration. The results presented are for a vibration frequency, $\omega_{w}=0.02$, and the distance from the wall has been normalized by the acoustic wavelength, $\lambda=2 \pi c_{0} / \omega_{w}$. The maximum pressure is apparently well predicted by Eq. (24) (dashed line). Note that, as predicted by Eq. (24), saturation occurs closer to the wall as the amplitude is increased. At the highest amplitude, shocking of the wave is immediate.

Bubbly shock waves are characterized by a pressure jump followed by oscillations, or ringing, at a frequency which, depending on the damping, varies from about a tenth of the postshock natural frequency to, for large damping, the postshock natural frequency for small damping. ${ }^{28}$ These oscillations are evident in the train of $N$ waves in Fig. 6, but they are highly damped due to the relatively large damping coefficient, $\delta_{D}=0.4$. In Fig. 7, the results for $A=0.005$ are replotted for several wavelengths of oscillation (away from the wall) along with a results for the same conditions except that the damping was lowered to 0.08 . While the ringing following the shocks is enhanced, these oscillations are merely superimposed on the basic $N$ wave predicted by the

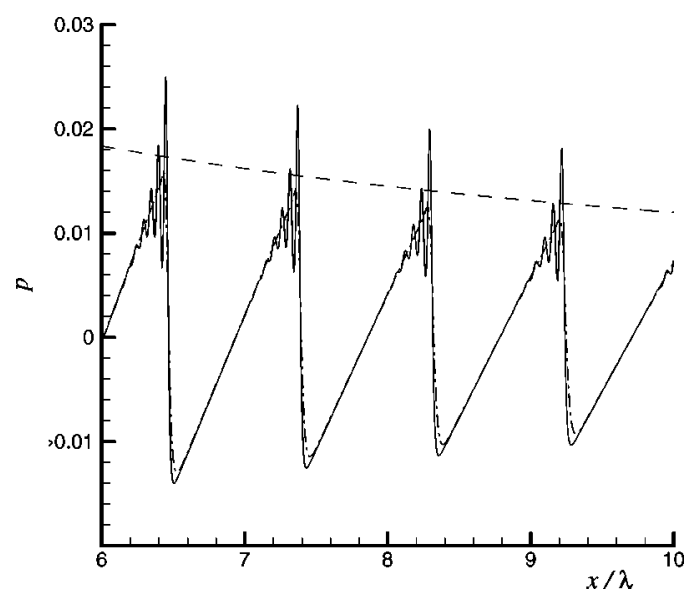

FIG. 7. Spatial evolution of (nondimensional) pressure for wall oscillation at $0.02 \omega_{0}$. Same conditions in Fig. 6 with $A=0.005$, and $\delta_{D}=0.4(-\cdot-)$ and $0.08(-)$.

process of acoustic saturation. This further suggests that the dissipation provided by the effective damping is asymptotically small in the evolution of the waves, with the majority of the dissipation due to the shock jump conditions. In other words, the collapses and rebounds following the shocks appear to have no dynamic significance for the evolution of the wave. For effective damping much larger than 0.4 , this is not true, as discussed previously. If the damping were set to zero then unattenuated ringing from each compression would persist indefinitely. On the other hand, as the frequency goes to zero the wavelength of the ringing following the shock becomes vanishingly small compared to the wavelength of the underlying $N$ wave. Thus acoustic saturation would take place for arbitrarily small damping, provided that the frequency is low enough.

At the higher frequency of $\omega_{w}=0.1$, as was shown in the Fig. 3, the amplitude of the radiated waves still saturates. However, comparison with the predicted maximum pressure of Eq. (24) becomes more difficult since collapse and rebound following collapse occupy a much larger fraction of the total cycle. This is shown in Fig. 8, where the spatial evolution of pressure is shown for $\omega_{w}=0.1$, for $A=0.005$ and several values of the effective damping. For comparison purposes, the result for $\omega_{w}=0.02$ and the prediction for the maximum pressure from Eq. (24) are replotted.

Thus the process of acoustic saturation in bubbly flow is, strictly speaking, only valid when the driving frequency is much lower than the bubble natural frequency, and when the damping is sufficiently small, but nonzero. van Wijngaarden ${ }^{36}$ noted that generally shock waves in bubbly flows involve an interplay between three mechanisms, convection (or wave steepening), dissipation, and dispersion. In going to higher frequencies, the interplay is complicated because not only is the ringing frequency following shocks closer to the driving frequency, but dispersive effects in Eq. (8) begin to become important. By experimentation, we find that the acoustic saturation process is valid for forcing frequencies below about $\omega_{w}=0.2$. 


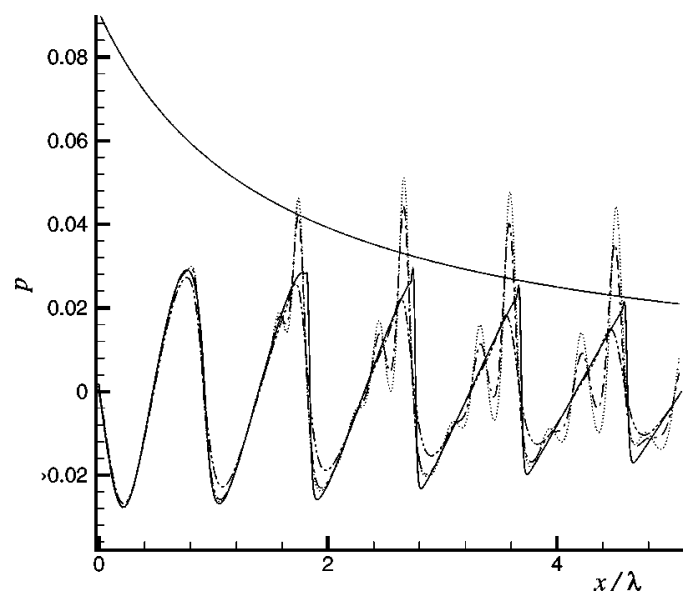

FIG. 8. Spatial evolution of (nondimensional) pressure for $\omega_{w}=0.1, A$ $=0.005$, and $\delta_{D}=0.4(-\cdot-), \delta_{D}=0.08(-\cdot-)$ and $\delta_{D}=0.04(\cdots)$. Also shown are results for $A=0.005$ and $\omega_{w}=0.02(-)$, as well as the prediction of Eq. (24).

\section{DISCUSSION}

We have computed numerically bubbly cavitation caused by the normal oscillating of a rigid wall. The principle result of the computations is that when the wall oscillation frequency is much smaller than the bubble natural frequency, the power radiated away from the wall is limited by an acoustic saturation effect, where the radiated power becomes independent of the amplitude of vibration. This is similar to the process which occurs in a pure gas, where nonlinear steepening of the generated waves leads to shocking of the wave train, and the dissipation associated with the shocks limits the radiated power. For low enough frequency, a formula for the maximum radiated pressure at a fixed distance from the wall was developed, based on weak shock theory, and found to be in excellent agreement with the computations. It was argued that the radiated waves are nearly independent of the viscosity (and indeed other dissipative mechanisms), for sufficiently small viscosity.

The process of acoustic saturation may have broader implications in cavitating flows which are driven at a frequency much lower than the bubble natural frequency. It has often been noted in experiments that as the cavitation number is decreased the noise first increases quickly, but increases at a lower rate or levels off or decreases with further decrease in the cavitation number. In experiments with submerged jets, for example, Franklin ${ }^{38,39}$ has noted an abrupt change in the noise generated by the jet as the cavitation number is decreased below a certain critical value. Below this value his data suggest a dependence of the mean-square pressure level in the far field of $1 / \sigma^{n}$, with $n$ around 5. It should be noted that the value of 5 is approximate, fitted with only a few data points, and, indeed subsequent measurements (e.g., Fig. 10 of Ref. 39) would seem to indicate a somewhat weaker dependence around $n=2$ to $n=3$. It has also been observed that below the critical cavitation number, the submerged jet flow exhibits a higher degree of organization, perhaps due to self-excitation of large scale structures by the acoustics, and that the acoustic field is more narrowly peaked about the dominant frequency.

We speculate that acoustic saturation may explain, at least in part, some of these observations. For spherical waves, the large $x$ expression in Eq. (24) remains valid except for a multiplicative logarithmic dependence on the radial distance from the source. ${ }^{40}$ For a fixed distance from the source, the average pressure level at saturation would, for low void fraction, obey the following scaling:

$$
\bar{p}^{2} \sim \frac{\left(p_{0}-p_{v}\right)^{3}}{\rho_{l} \alpha_{0} \omega^{2} x^{2}},
$$

where $\omega$ is the (dimensional) frequency of the source. This follows from Eq. (24) at large $x$, using definitions from Sec. III. For submerged jets, Franklin ${ }^{38}$ suggests that the peak Strouhal number of the radiated noise, $\mathrm{St}=f D / U$ scales like $\sigma^{2}$, where $D$ is the jet diameter and $U$ the jet velocity, and the cavitation number is defined with the jet velocity. Noting that Franklin obtained different cavitation numbers by varying the jet velocity while holding the pressure constant, we obtain, again at a fixed location,

$$
\bar{p}^{2} \sim \frac{\left(p_{0}-p_{v}\right)^{2}}{\alpha_{0} \sigma^{3}},
$$

in agreement with his observations of submerged jets.

Of course, this result is open to criticism on several fronts. The result here is for a simple harmonic source, not a turbulent bubbly flow with a broad band spectra. Also, we have not accounted for the effects of having a distribution of bubble sizes, and gradients in bubble concentrations. Nevertheless, it appears possible that the acoustic saturation process could exist in practical cavitating flows, and merits further investigation.

\section{ACKNOWLEDGMENT}

C.E.B. acknowledges the support of the Office of Naval Research under Grant No. N00014-91-J-1295.

${ }^{1}$ A. T. Preston, T. Colonius, and C. E. Brennen, "Bubbly cavitating flow in a nozzle" (unpublished).

${ }^{2} \mathrm{~L}$. van Wijngaarden, "On the collective collapse of a large number of gas bubbles in water," in Proceedings of the 11th International Conference on Applied Mechanics (Springer, Berlin, 1964), pp. 854-861.

${ }^{3}$ L. d'Agostino and C. E. Brennen, "On the acoustical dynamics of bubble clouds," in ASME Cavitation and Multiphase Flow Forum, 1983 (unpublished), pp. 72-75.

${ }^{4}$ L. D'Agostino and C. E. Brennen, "Acoustical absorption and scattering cross-sections of spherical bubble clouds," J. Acoust. Soc. Am. 84, 2126 (1988).

${ }^{5}$ L. D'Agostino and C. E. Brennen, "Linearized dynamics of spherical bubble clouds,' J. Fluid Mech. 199, 155 (1989).

${ }^{6} \mathrm{R}$. Omta, "Oscillations of a cloud of bubbles of small and not so small amplitude,'” J. Acoust. Soc. Am. 82, 1018 (1987).

${ }^{7}$ A. Prosperetti, "Bubble-related ambient noise in the ocean," J. Acoust. Soc. Am. 84, 1042 (1988).

${ }^{8}$ S. Kumar and C. E. Brennen, "Non-linear effects in the dynamics of clouds of bubbles," J. Acoust. Soc. Am. 89, 707 (1991).

${ }^{9}$ S. Kumar and C. E. Brennen, "Harmonic cascading in bubble clouds," in Proceedings of the International Symposium on Propulsors and Cavitation, Hamburg, 1992 (unpublished), pp. 171-179.

${ }^{10}$ S. Kumar and C. E. Brennen, "Some nonlinear interactive effects in bubbly cavitating clouds," J. Fluid Mech. 253, 565 (1993).

${ }^{11}$ C. S. Song, "Direct numerical simulation of sheet cavitation and cloud 
cavitation," in 1997 ONR Workshop on the Dynamics of Bubbly Flows, San Diego, CA, 1997 (unpublished).

${ }^{12}$ C. L. Merkle and J. Feng, "Dynamics of sheet cavitation," in 1998 ONR Workshop on Free Surface Turbulence and Bubbly Flows, Pasadena, CA, 1998 (unpublished).

${ }^{13}$ M. Kameda, N. Shimaura, F. Higashino, and V. Matsumoto, "Shock waves in a uniform bubbly flow," Phys. Fluids 10, 2661 (1998).

${ }^{14}$ M. Kameda and Y. Matsumoto, "Shock waves in a liquid containing small gas bubbles," Phys. Fluids 8, 322 (1996).

${ }^{15}$ M. Rein and G. E. A. Meier, "On the influence of different parameters on heterogeneous shock cavitation," J. Acoust. Soc. Am. 88, 1921 (1990).

${ }^{16} \mathrm{M}$. Rein and G. E. A. Meier, "On the dynamics of heterogeneous shock cavitation," Acustica 71, 1 (1990).

${ }^{17} \mathrm{P}$. Smereka and S. Banerjee, "The dynamics of periodically driven bubble clouds," Phys. Fluids 31, 3519 (1988).

${ }^{18}$ Y.-C. Wang and C. E. Brennen, "Shock wave development in the collapse of a cloud of bubbles," in ASME Cavitaiton and Multiphase Flow Forum, No. FED-194, 1994 (unpublished), pp. 15-20.

${ }^{19}$ Y.-C. Wang and C. E. Brennen, "The noise generated by the collapse of a cloud of cavitation bubbles," in ASME/JSME Symp. on Cavitation and Gas-Liquid Flow in Fluid Machinery and Devices, No. FED-226, 1995 (unpublished), pp. 17-29.

${ }^{20}$ Y.-C. Wang and C. E. Brennen, "Shock wave and noise in the collapse of a cloud of cavitation bubbles," in Proceedings of the 20th International Symposium on Shock Waves, Pasadena, CA, 1995 (unpublished), pp. $1213-1218$.

${ }^{21}$ A. Kubota, H. Kato, and H. Yamaguchi, "A new modelling of cavitating flows: A numerical study of unsteady cavitation on a hydrofoil section," J. Fluid Mech. 240, 59 (1992).

${ }^{22}$ L. D'Agostino, C. Brennen, and A. Acosta, "Linearized dynamics of twodimensional bubbly and cavitating flows over slender surfaces," J. Fluid Mech. 192, 485 (1988).

${ }^{23}$ A. Biesheuvel and L. Van Wijngaarden, "Two-phase flow equations for a dilute dispersion of gas bubbles in liquid," J. Fluid Mech. 148, 301 (1984).
${ }^{24}$ A. Prosperetti, L. A. Crum, and K. W. Commander, "Nonlinear bubble dynamics,"' J. Acoust. Soc. Am. 83, 502 (1988).

${ }^{25}$ D. Z. Zhang and A. Prosperetti, "Ensemble phase-averaged equations for bubbly flows," Phys. Fluids 6, 2956 (1994).

${ }^{26}$ D. Givoli, “Non-reflecting boundary conditions," J. Comput. Phys. 94, 1 (1991).

${ }^{27}$ S. V. Tsynkov, "Numerical solution of problems on unbounded domains," Appl. Numer. Math. 27, 465 (1998).

${ }^{28}$ C. E. Brennen, Cavitation and Bubble Dynamics (Oxford University Press, Oxford, 1995).

${ }^{29}$ Y. Wang, "Shock waves in bubbly cavitating flows," Ph.D. thesis, California Institute of Technology, 1996.

${ }^{30}$ E. Hairer and G. Wanner, Solving Ordinary Differential Equations II, Revised ed. (Springer, New York, 1996).

${ }^{31}$ J. H. Ferziger, Numerical Methods for Engineering Application (Wiley, New York, 1981).

${ }^{32}$ R. Chapman and M. Plesset, "'Oscillations of a cloud of bubbles of small and not so small amplitude," ASME J. Basic Eng. 93, 373 (1971).

${ }^{33}$ M. Watanabe and A. Prosperetti, "Shock waves in dilute bubbly liquids," J. Fluid Mech. 274, 349 (1994).

${ }^{34}$ Y. Hao and A. Prosperetti, "The dynamics of vapor bubbles in acoustic pressure fields,"' Phys. Fluids 11, 2008 (1999).

${ }^{35}$ Y.-C. Wang, "Stability analysis of one dimensional steady cavitating nozzle flows with bubble size distribution," submitted to J. Fluids Eng.

${ }^{36} \mathrm{~L}$. van Wijngaarden, "One-dimensional flow of liquids containing small gas bubbles," Annu. Rev. Fluid Mech. 4, 369 (1972).

${ }^{37}$ A. D. Pierce, Acoustics (Acoustical Society of America, New York, 1989).

${ }^{38}$ R. E. Franklin, Jet Cavitation, in Jets and Cavities - International Symposium Vol. 31 (ASME-Fluids Engineering Division, New York, 1985).

${ }^{39}$ R. E. Franklin, Cavitation in the Submerged Jet: The Effects of Ambient Pressure and Total Gas Content," in International Symposium on Cavitation Noise and Erosion in Fluid Systems Vol. 88 (ASME-Fluids Engineering Division, New York, 1989).

${ }^{40}$ J. A. Shooter, T. G. Muir, and D. T. Blackstock, "Acoustic saturation of spherical waves in water,” J. Acoust. Soc. Am. 55, 54 (1974). 